\title{
Estimación del Perfil de Morbilidad en el Personal de la Aviación Civil en Colombia
}

\section{Estimating the morbidity profile amongst Colombian civil aviation personnel}

\author{
María A. Salamanca y Hugo A. Fajardo \\ Unidad de Medicina Aeroespacial, Universidad Nacional de Colombia. Bogotá. angelita764@yahoo.com, \\ hafajardor@unal.edu.co \\ Recibido 1 Octubre 2008/Enviado para Modificación 10 Abril 2009/Aceptado 5 Mayo 2009

\section{RESUMEN}

Objetivo Describir los principales procesos de morbilidad en tripulaciones y controladores aéreos pertenecientes a la aviación civil de Colombia.

Métodos Estudio transversal descriptivo. Se tomó una muestra aleatoria de todos los registros clínicos existentes en el archivo de la dirección de Medicina de Aviación de la autoridad de la Aeronáutica Civil de Colombia que correspondió a 1059 registros (de pilotos, auxiliares de senvicios a bordo, controladores aéreos y operadores de estación aérea). Se revisaron las historias clínicas y se tomaron los datos de identificación, antecedentes, morbilidad y limitaciones actuales.

Resultados De los 1059 registros, 68,2\% correspondieron a hombres, el promedio de edad fue 37 años. El 22,9\% refirieron algún antecedente médico en la primera certificación. En el 17,1 \% se registró consumo de alcohol y en el 11,9 \% tabaquismo. Los principales diagnósticos encontrados fueron: los trastornos de la vision (52,2\%), las dislipidemias $(40,6 \%)$, aumento de peso $(37,5 \%)$ principalmente sobrepeso $(33,3 \%)$, la pérdida auditiva $(22,0 \%)$, sano $(14,0 \%)$ y HTA $(8,1 \%)$. Los diagnósticos se conservaron en los diferentes grupos aunque en diferente orden.

Conclusiones En otros estudios predominan las enfermedades cardiovasculares entre tripulantes y controladores aéreos. En Colombia los trastornos de la visión fueron los predominantes. No sabemos si hay una predisposición a padecer de estas enfermedades. En segundo lugar se encontraron factores de riesgo cardiovascular lo cual reviste vital importancia para la implementación de programas de promoción y prevención y para la salud publica.

Palabras Clave: Morbilidad, aviación, sobrepeso, dislipidemias, trastornos de la visión, pérdida auditiva (fuente: DeCS, BIREME).

\section{ABSTRACT}

Objective Describing main morbidity rate amongst Colombian civil aviation cockpit and air traffic control personnel.

Methods This was a cross-sectional study. A random sample of 1,059 records (pilots, flight attendants, air traffic controllers) was taken from Colombian aeronautical authority files. Identification, background, morbidity and limitation data was reviewed for each one. 
Results $68,2 \%$ of the records concerned males. Average age was 37. 22,9\% of the sample had some medical antecedent recorded on their first certification. Alcohol consumption (occasional) was recorded for $17,1 \%$ of the sample and $11,9 \%$ were smokers. The main diagnoses made were: vision disorders $(52,2 \%)$, dyslipidemias $(40,6 \%)$, being overweight $(37,5 \%)$, having hearing loss $(22,0 \%)$, being healthy $(14,0$ $\%)$ and suffering from hypertension (8,1\%). Each group had the same diagnoses, but occurring in a different order.

Conclusions: Others studies have found cardiovascular disease to be the main cause of morbidity amongst cockpit and air traffic control personnel. Vision disorders were the main disorder occurring in Colombia. We do not know if there is a predisposition amongst Colombians to suffer such morbidity. Cardiovascular risk factors were found in second place in Colombia, meaning that public health prevention and promotion countermeasures should be taken.

Key Words: Aviation, morbidity, overweight, dyslipidemia, vision disorder, hearing loss (source: $\mathrm{MeSH}, \mathrm{NLM}$ ).

$\mathrm{A}$

lrededor del mundo se han realizado estudios en procura de acercarse al perfil de morbilidad y mortalidad del personal de tripulación y de apoyo al vuelo en tierra, los cuales presentan limitaciones en cuanto a las fuentes de información. Sin embargo, se ha establecido que la prevalencia global de morbilidad suele ser menor entre este personal posiblemente debido a los procesos de certificación y chequeos médicos periódicos con lo cual se disminuye también la mortalidad (1,3-6,11). Las patologías crónicas más prevalentes entre este personal son las cardiovasculares y de ellas especialmente la HTA, así mismo los defectos visuales refractivos (4-6,11). Estudios más específicos han encontrado mayor prevalencia de e incidencia de algunos tipos de cáncer en personal de tripulación (cáncer de piel, de seno, de cerebro) en comparación con la población general $(1,2,12)$; en cuanto a mortalidad los accidentes aéreos son una causa mucho mayor de muerte para el personal de tripulación que para la población general así como el SIDA, según estudios europeos $(1,2,8,9,13)$.

Existen pocos estudios latinoamericanos de morbimortalidad publicados. El presente trabajo intenta un acercamiento al perfil de morbilidad en el personal aeronáutico civil activo de Colombia con el fin de tener alguna herramienta que permita tomar medidas más cercanas a la realidad así como desarrollar otros estudios que profundicen problemáticas de particular interés para Iberoamérica. 


\section{MÉTODO}

Es un estudio transversal descriptivo incluyendo como población de estudio al personal certificado medicamente por Aerocivil de Colombia, y cuya certificación estuviera vigente al 10 de marzo de 2005.

Se tomaron los registros médicos los cuales fueron seleccionados en forma aleatoria y se extrajo la siguiente información:

Identificación, antecedentes médicos, morbilidad y probables limitaciones o incapacidades o secuelas. Los datos se consignaron en el programa Epi-info versión 3.3.2, siguiendo los criterios diagnósticos establecidos internacionalmente.

La muestra se tomó por conglomerados entre cada uno de los grupos o clases de certificación aeromedica, tomando como prevalencia el $50 \%$, asumiendo el peor escenario ya que en Colombia no existen estudios previos. Se tomó un nivel de confianza del $95 \%$ o valor $\mathrm{p}<=0,05$.

$\mathrm{Al}$ realizar el análisis univariado se obtuvieron promedios y proporciones en las variables correspondientes como medidas de resumen y dispersión y se establecieron asociaciones o correlaciones en el análisis bivariado, utilizando Epi-info versión 3.3.2, se realizaron gráficos y tablas respectivas. Se obtuvo el consentimiento institucional y el presente estudio no tiene conflicto de intereses

\section{RESULTADOS}

Del personal que tiene certificación médica aeronáutica vigente a marzo 10 de 2005, que totalizaba 8186 , se tomó una muestra total de 1059 historias clínicas. El promedio de edad en toda la muestra fue 37 años, con un rango entre 19 y 91 años y predominio del sexo masculino, 68,2 \%.

El 69,4\% de la muestra no registró limitaciones específicas en el certificado médico más recientemente expedido; las limitaciones más frecuentes fueron el uso de lentes $(23,3 \%)$, uso de protector auditivo $(10,1 \%)$ y control de alguna alteración metabólica (2,8 \%). En ningún registro se reportó consumo de sustancias ilegales. En la Tabla 3 se resume la morbilidad encontrada. 
Grupo de Auxiliares de Servicios a Bordo (ASA)

Los diagnósticos más frecuentes registrados en este grupo al ingreso fueron los trastornos oculares refractivos, de ellos principalmente el astigmatismo en el $16,9 \%$. Luego la condición sano y en tercer lugar las dislipidemias, principalmente la hipercolesterolemia en el 1,8\%.

Entre la primera y última valoración se encontraron diferentes trastornos cuya prevalencia es variable en el tiempo debido a que algunos se resuelven y otros no. Las infecciones respiratorias altas fueron reportadas en algún momento en el 40,2 \% de este grupo. Las dislipidemias en el 25,7 \%, predominando la hipercolesterolemia. Y los trastornos oculares refractivos en el 25,1\%, especialmente el astigmatismo.

La proporción de auxiliares de servicios a bordo que reportaron lesiones osteomusculares durante el periodo comprendido entre la primera certificación aeromedica y el 10 de marzo de 2005 fue de 20,7 \%. Lo cual difiere de los demás grupos.

En cuanto a la morbilidad actual la mayor prevalencia la tienen los trastornos oculares refractivos, predominando el astigmatismo en el 25,7 \%. Luego la condición sano y en tercer lugar las dislipidemias, predominando la hipercolesterolemia $(9,8 \%)$.

\section{Grupo de Controladores de Tránsito Aéreo (ATC)}

Los diagnósticos más frecuentes registrados en este grupo al ingreso fueron (Tabla 1) la condición sano; luego los trastornos oculares refractivos predominando el astigmatismo en el 16,0 \%; y en tercer lugar el exceso de peso, principalmente sobrepeso en $18,4 \%$.

Entre la primera y última valoración se encontraron los siguientes trastornos: dislipidemias $(55,2 \%)$ en especial la hipertrigliceridemia ( $26,9 \%)$; trastornos oculares refractivos (42\%) especialmente el astigmatismo (19,8\%); y exceso de peso (34,9\%), fundamentalmente el sobrepeso $(31,1 \%)$.

La principal morbilidad actual en controladores de tránsito aéreo es por (Tabla 1) trastornos oculares refractivos, especialmente el astigmatismo $(18,9 \%)$ y la miopía $(18,9 \%)$; exceso de peso, predominando el sobrepeso $(41,5 \%)$ y las dislipidemias. 
Grupo de Operadores de Estación Aérea (OEA)

Los diagnósticos más frecuentes registrados en este grupo al ingreso fueron (Tabla 1) los trastornos oculares refractivos, principalmente el astigmatismo y la miopía con 14,1 \% cada uno; las dislipidemias, predominando la hipertrigliceridemia $(21,7 \%)$; y el exceso de peso con predominio del sobrepeso $(31,5 \%)$.

Entre la primera y última valoración la mayoría de operadores de estación aérea no reportaron trastornos nuevos $(51,1 \%)$. El $12 \%$ registró dislipidemias, y el $10,9 \%$ trastornos oculares refractivos.

La principal morbilidad actual en operadores de estación aérea es por (Tabla 1) trastornos oculares refractivos con predominio del astigmatismo en el 20,7 \%; las dislipidemias, principalmente la hipertrigliceridemia en el $21,7 \%$ y en tercer lugar el exceso de peso, predominando el sobrepeso $(31,5 \%)$.

Tabla 1. Diagnósticos más frecuentes en los diferentes momentos de la certificación médica desde la primera expedición hasta la más reciente

\begin{tabular}{|c|c|c|c|c|c|c|c|c|c|c|c|}
\hline \multirow[t]{2}{*}{ Grupo } & \multicolumn{2}{|c|}{$\begin{array}{l}\text { Sano } \\
(\%)\end{array}$} & \multicolumn{3}{|c|}{$\begin{array}{c}\text { Trastornos oculares refractivos } \\
(\%)\end{array}$} & \multicolumn{3}{|c|}{$\begin{array}{l}\text { Dislipidemias } \\
(\%)\end{array}$} & \multicolumn{3}{|c|}{$\begin{array}{c}\text { Exceso de peso } \\
(\%)\end{array}$} \\
\hline & $\begin{array}{l}\text { prev. } \\
\text { ingreso }\end{array}$ & $\begin{array}{l}\text { prev. } \\
\text { Actual }\end{array}$ & $\begin{array}{l}\text { prev. } \\
\text { ingreso }\end{array}$ & $\begin{array}{l}\text { proporción } \\
\text { incidencia }\end{array}$ & $\begin{array}{l}\text { prev. } \\
\text { actual }\end{array}$ & $\begin{array}{l}\text { prev. } \\
\text { ingreso }\end{array}$ & $\begin{array}{l}\text { proporción } \\
\text { incidencia }\end{array}$ & $\begin{array}{l}\text { prev. } \\
\text { actual }\end{array}$ & $\begin{array}{l}\text { prev. } \\
\text { ingreso }\end{array}$ & $\begin{array}{l}\text { proporción } \\
\text { incidencia }\end{array}$ & $\begin{array}{l}\text { prev. } \\
\text { actual }\end{array}$ \\
\hline Pilotos & 43,6 & 9,1 & 19,7 & 31,4 & 51,1 & 16,8 & 36,2 & 53,0 & 13,4 & 36,7 & 50,1 \\
\hline ASA & 38,8 & 24,9 & 46,7 & 4,8 & 51,5 & 13,0 & 10,7 & 23,7 & 2,7 & 44,9 & 47,6 \\
\hline ATC & 33,5 & 26,4 & 29,7 & 13,8 & 43,5 & 12,8 & 20,2 & 33 & 18,9 & 28,7 & 47,6 \\
\hline OEA & 8,7 & 7,6 & 42,4 & 14,1 & 56,5 & 39,1 & 7,6 & 46,7 & 37,0 & 0 & 37,0 \\
\hline
\end{tabular}

Grupo de Pilotos

De 4734 pilotos se seleccionaron aleatoriamente 417 registros, proporcionalmente para cada grupo de pilotos (pilotos privados, pilotos comerciales, pilotos de helicóptero y pilotos de transporte de línea). Los diagnósticos más frecuentes registrados en este grupo al ingreso fueron (Tabla 1) la condición sano, luego los trastornos oculares refractivos, de los cuales se destaca la miopía como principal trastorno con el 8,6 $\%$; y en tercer lugar las dislipidemias y de ellas principalmente la hipertrigliceridemia en el $7,4 \%$.

Entre la primera y última valoración se encontraron los siguientes trastornos: las dislipidemias $(69,5 \%)$ y de ellas en especial la dislipidemia mixta (36,7\%), seguido 
del exceso de peso en el 48,2 \%, predominantemente el sobrepeso (45,1\%); y las alteraciones oculares refractivas $(44,6 \%)$ especialmente la presbicia $(21,6 \%)$.

La principal morbilidad actual en pilotos es por (ver tabla 1): las dislipidemias, especialmente la hipertrigliceridemia en el 20,6\%, los trastornos oculares refractivos, con predominio de la presbicia en el $17,7 \%$ y el exceso de peso, con sobrepeso en el $44,1 \%$.

\section{DISCUSIÓN}

En Colombia las patologías más frecuentes entre el personal de la aviación civil son las relacionadas con factores de riesgo cardiovascular y los defectos visuales refractivos similar a lo reportado en otros estudios, sin embargo, no se encontró alta prevalencia en otras enfermedades como los cálculos renales y el cáncer.

Por otra parte, el exceso de peso es una patología frecuente entre el personal de la aviación civil en Colombia concordante con otros estudios donde el exceso de peso en pilotos es un problema.

En relación específicamente al personal de auxiliares de servicios a bordo (ASA) los resultados obtenidos en este estudio de los registros de incapacidades laborales de este personal evidencian una alta incidencia de infecciones respiratorias altas lo cual es acorde con estudios de otros países. No se encontraron alteraciones relacionadas con el ciclo menstrual $(7,10,14)$.

Aún no es posible establecer otros diagnósticos como la infección por VIH ya que este requiere practicar la prueba previa autorización individual y es de carácter confidencial, por ello es esperable que ni sea realizada ni sea reportada durante la certificación médica.

Entre el grupo de controladores de tránsito aéreo y operadores de estación aeronáutica la HTAno fue tan frecuente como en otros estudios (6). En cambio los trastornos psiquiátricos fueron más incidentes que en el grupo de controladores aéreos sin alcanzar a figurar dentro de los más importantes. En un controlador aéreo se encontraron alteraciones del sueño.

Evidentemente, el presente estudio tuvo importantes limitaciones inherentes a la revisión de archivo, información incompleta y autorreporte. Además de los 
registros no se puede obtener información de estrato socioeconómico, raza, nacionalidad; antecedentes ginecoobstetricos; horas de vuelo de ASA y de IDV.

También la variación de los criterios diagnósticos a través del tiempo son una importante limitante pues estos han cambiado para diversas patologías y es esa una de las posibles razones para que no coincidan ciertas prevalencias e incidencias con estudios realizados en otras regiones del mundo.

En conclusión podemos afirmar que a pesar de las limitaciones mencionadas este es el primer estudio en Colombia que se acerca de manera objetiva al perfil de morbilidad de la población civil que requiere certificación médica para desempeñar sus actividades laborales en el medio aeronáutico.

Se requiere implementar bases de datos en la salud pública colombiana y la realización de estudios más específicos para hacer un mejor acercamiento a la morbilidad del personal de la aviación civil

\section{REFERENCIAS}

1. Ballard T, Lagorio S, De Angelis G, Verdecchia A. Cancer Incidence and Mortality Among Flight Personnel: A Meta-Analysis. Aviat Space Environ Med 2000; 71: 216-24.

2. Band P, Spinelli JJ, Ng VT, Moody J, Gallagher RP. Mortality and cancer incidence in a cohort of commercial airline pilots. Aviat Space Environ Med 1990; 61:299-302.

3. Benesh L. Occupational Medicine in the Aviation Industry. Int J Occup Med 1962; 4 (7): 370-2.

4. Booze Ch F. Prevalence of selected pathology among currently certified active airmen Technical Report FAA; Apr. 1981.

5. Booze Ch F. Prevalence of disease among active civil airmen. Technical Report FAA; 1990.

6. Booze Ch F. The morbidity experience of Air Traffic Control Personnel.Technical Report FAA; 1978.

7. Iglesias R, Terrés A, Chavarria A. Disorders of the Menstrual Cycle in Airline Stewardesses. Aviat Space Environ Med 1980; 51: 518-20.

8. Irvine D, Davies DM. The mortality of British Airways pilots, 1966-1989: a proportional mortality study. Aviat Space Environ Med 1992; 63:276-9.

9. Kaji MTT, Asukata I, Tajima N, Yamamoto K, Yamamoto Y, et al. Mortality Experience of cockpit crewmembers from Japan Airlines. Aviat Space Environ Med 1993; 64:748-750.

10. Nagda NL, Koontz MD. Review of studies on Flight Attendant Health and Comfort in Airliner Cabins. Aviat Space Environ Med 2003; 74:101-9.

11. Parker P, Stepp RJ, Snyder QC. Morbidity among Airline Pilots: The AMAS Experience. Aviat Space Environ Med 2001; 72:816-820.

12. Pukkala E, Aspholm R, Auvinen A, Eliasch $H$, Gundestrup $M$, Haldorsen $T$, et al. Incidence of cancer among Nordic airline pilots over five decades: occupational cohort study BMJ 2002;325: 567-571.

13. Salisbury DA, Band PR, Threlfall WJ, Gallagher RP. Mortality among British Columbia pilots. Aviat Space Environ Med 1991;62:351-2.

14. Whelan EA, Lawson CC, Grajewski B, Petersen MR, Pinkerton LE, Ward EM, et al. Prevalence of Respiratory Symptoms among female Flight Attendants and Teachers. Occup Environ Med 2003; 62:929-34. 